\title{
DIAGNOSTIC VALUE OF ULTRASONOGRAPHY IN DETECTION OF METASTATIC INTERNAL MAMMARY LYMPHADENOPATHY IN BREAST CANCER PATIENTS
}

Alaa Eldin Mohamed Abd Elhamid Mostafa, Mohamed Mahmoud Elshafey, Rasha Omar Elsaka,* Nesreen Mesbah Elsayed Ghazy

Department of Radiodiagnosis, Department of Clinical Oncology and Nuclear Medicine,* Faculty of Medicine, University of Alexandria.

Lymphatic drainage of breast to which breast cancer can metastasize includes the
axillary (ALN), internal mammary (IMLN), infraclavicular, or supraclavicular nodes.
3-12\% of breast lymphatics drain to IM chain.
Evaluation of regional lymph node status (N) is crucial for breast cancer disease
staging, treatment planning, and prognosis. It is crucial to identify IMLN metastases;
as if present it not only changes the nodal stage (it upstages the patient to clinical stage
III disease at minimum) and prognosis of the patient but also affects the planning of
radiation therapy.
The independent risk factors that increase likelihood of IMLN metastasis include
medial tumor location, large tumor size, more involved ALNs, age < 35 years, higher
tumor grade, tumor with calcification, nipple inversion, triple-negative hormone
receptor status, lymphovascular invasion and negative HER2 status.
Evaluation of the internal mammary nodes with parasternal real-time handheld US is a
cost effective and convenient way to detect and upstage nodal metastases.

\section{AMM OF THE WORK}

The aim of this study was to assess the diagnostic value of parasternal US in detection of IMLN in breast cancer patients.

\section{PATIENTS AND METHODS}

This study was conducted on 50 female patients with proven imaging diagnosis of breast cancer referred to breast diagnostic imaging units of Shark El-Madina Hospital and Alexandria Main University Hospital, for parasternal ultrasonography of IMLN

All patients were subjected to the following scheme upon referral to diagnostic breast imaging unit: A focused history, breast physical examination, Laboratory breast imaging unit: A focused history, breast physical examination, Laboratory
assessment.

Imaging evaluation including: Breast mammography, whole breast Ultrasound with extended parasternal Ultrasound of parasternal region bilaterally, computed tomography of chest, histopathological assessment of primary breast lesion and IMLN whenever achievable.

Revision of other confirmatory imaging techniques (MRI, PET/CT) and Follow up imaging data after therapy whenever available.
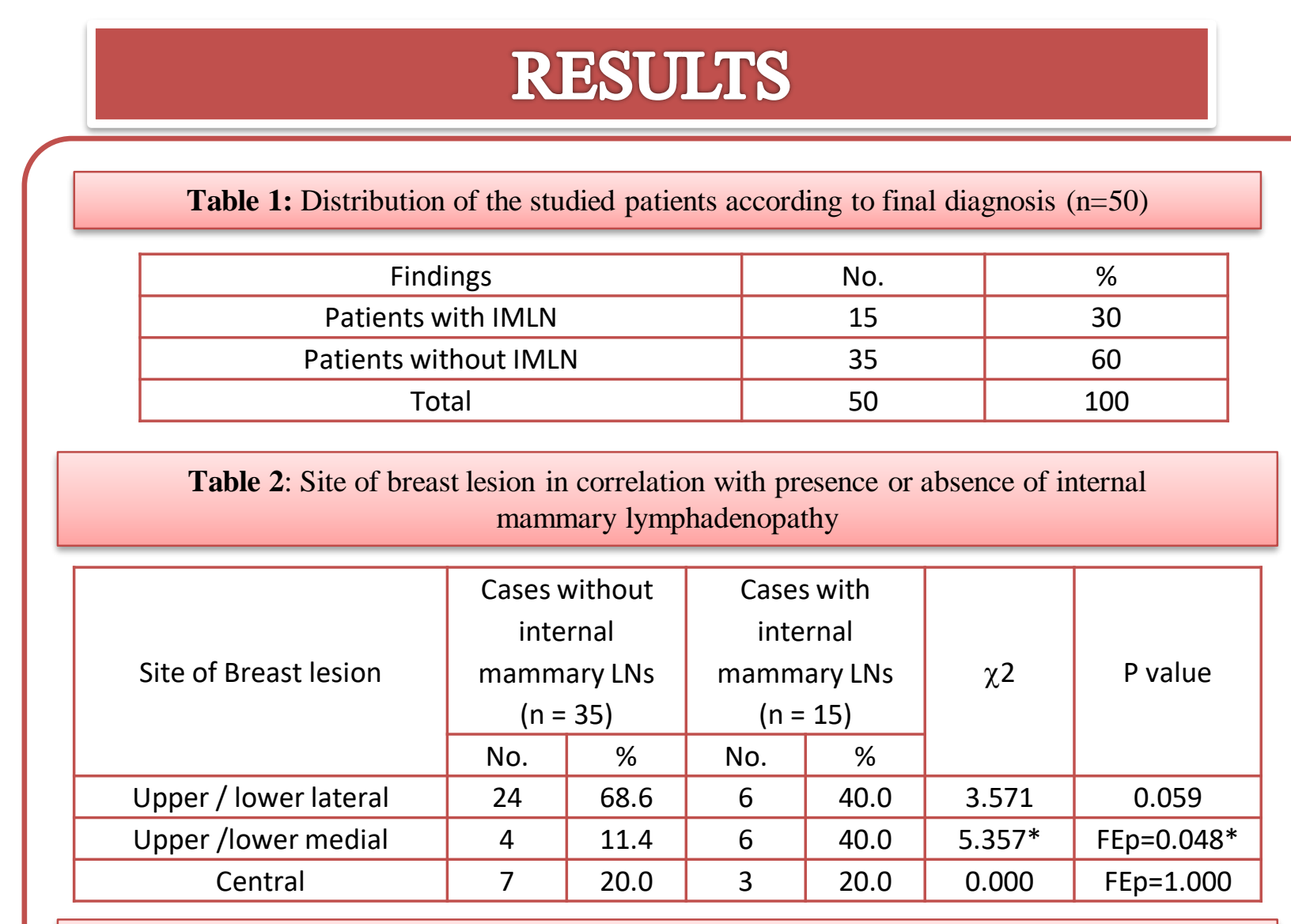
Table 3: Presence of concomitant malignant axillary lymphadenopathy in correlation to presence
or abscence of internal mammary lymphadenopathy.

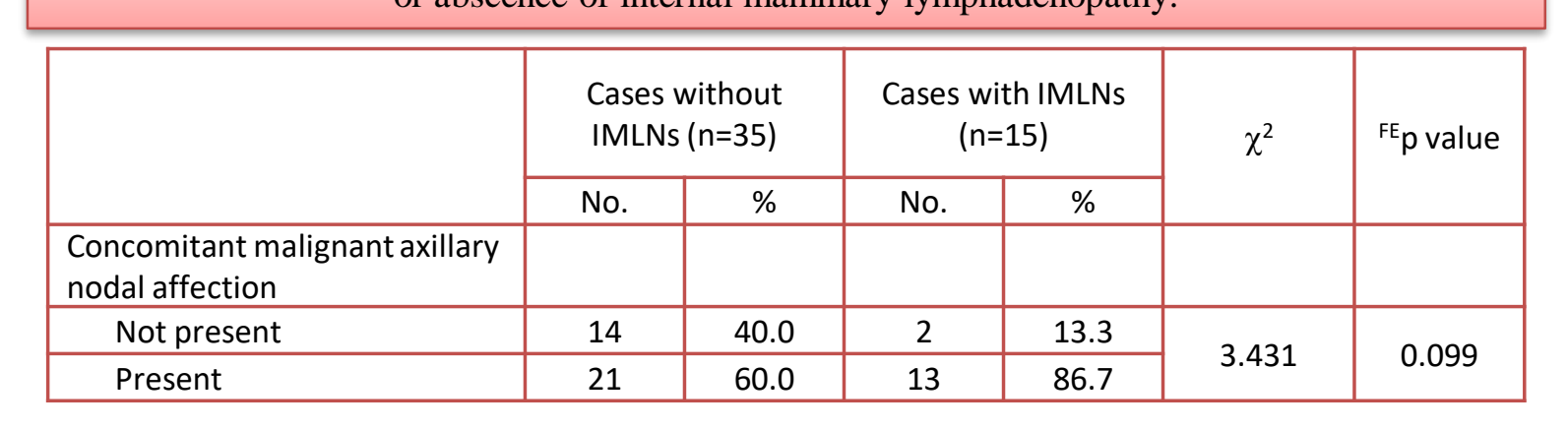

Table 4: Diagnostic yield of ultrasonography in detection of IMLN in 50 patients correlated to
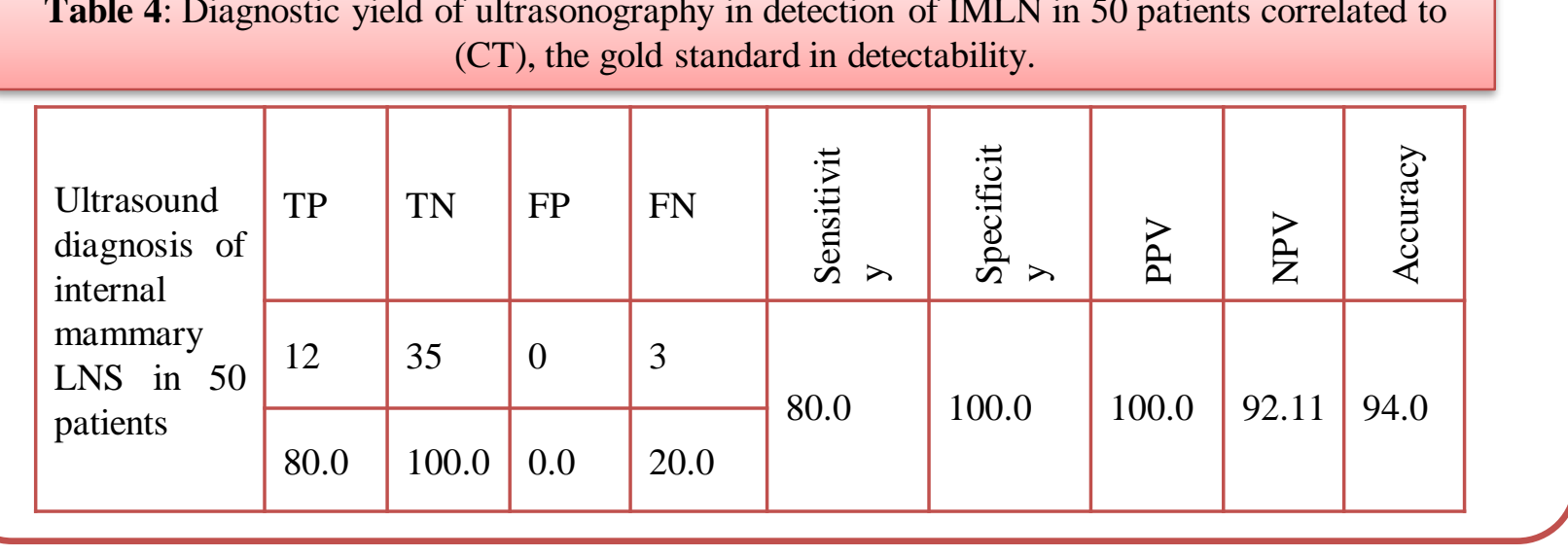
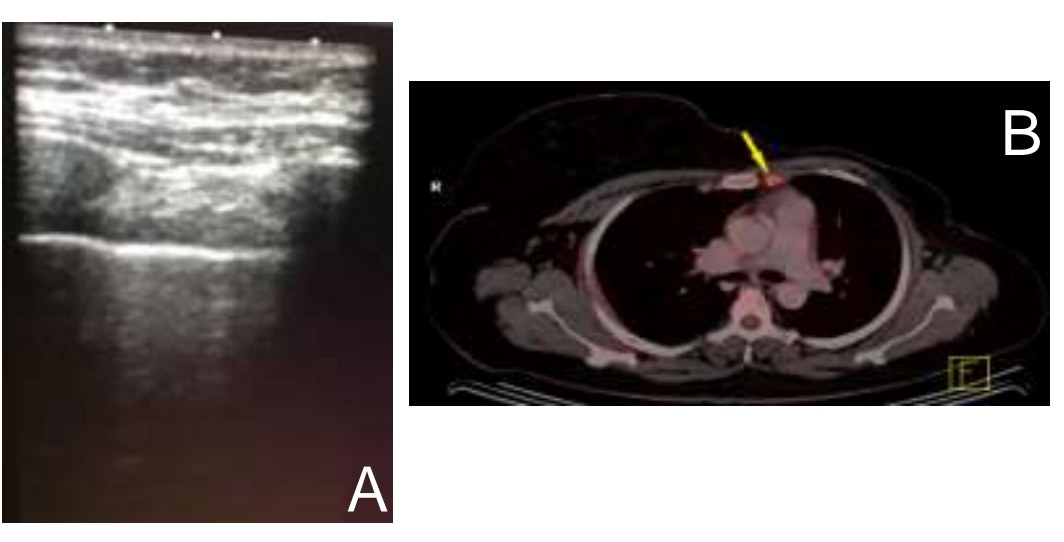

Figure 1: False negative US diagnosis of Left IMLN in a patient treated for left breast cancer with positive metastatic
A) US image : no IMLN

B) PET/CT fusion image: left IMLN (yellow arrow).

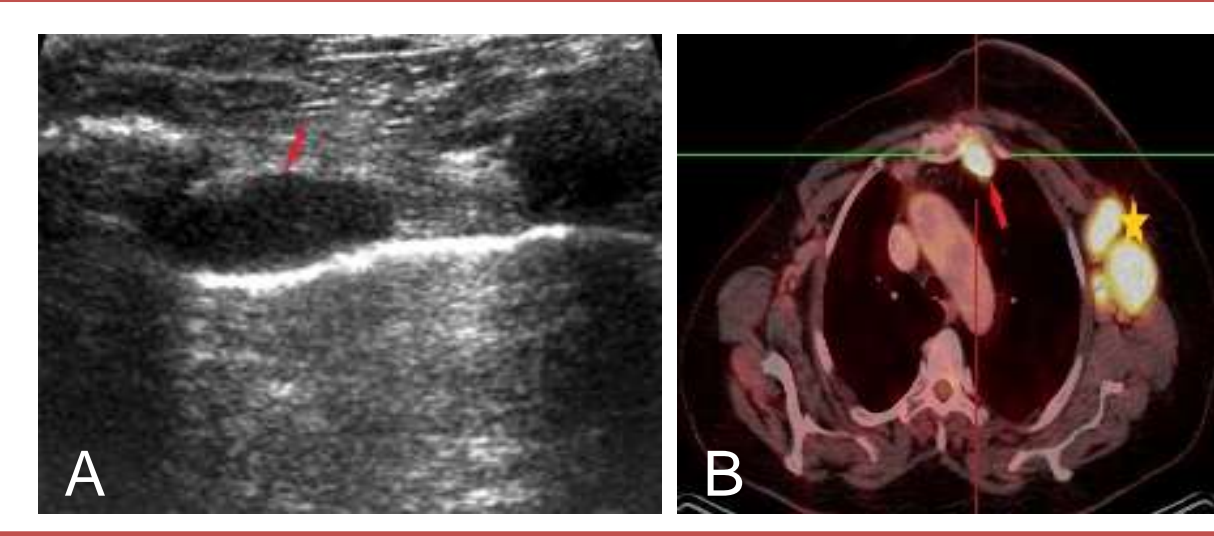

Figure 2: True positive US diagnosis of left IMLN in a patien
diagnosed as metastatic left axillary and left IMLN by PET/CT.

right breast cancer

\section{CONCLUSION}

- Ultrasonography (US) is a cost effective, convenient and easily accessible method in detection of IMLN with high accuracy ( $94 \%$ ) with no risk of radiation as compared to CT. so parasternal US should screening for breast cancer.

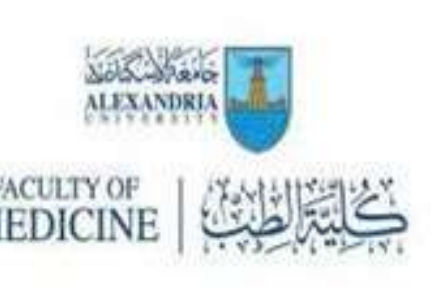
2021OAlexandria Faculty of Medicine
CC-BY-NC 